\title{
TUMOR DE MAMA FILOIDE MALIGNO COM COMPONENTE SARCOMATOSO: REVISÃO INTEGRATIVA
}

\author{
MALIGNO FILOID BREAST TUMOR WITH SARCOMATOUS \\ COMPONENT: INTEGRATIVE REVIEW
}

Liliane de Lima Caldas Gervásio ${ }^{1}$ Thais Emanuele Garrido Torres ${ }^{2}$

Macerlane de Lira Silva ${ }^{3}$

Elisângela Vilar de Assis ${ }^{4}$

Ankilma do Nascimento Andrade Feitosa ${ }^{5}$

\begin{abstract}
RESUMO: Introdução: o tumor filoide (TF) da mama é uma neoplasia fibroepitelial, responsável por menos de $1 \%$ dos tumores de mama. É o único tumor benigno da mama em $80 \%$ dos casos, capaz de malignar-se, o que ocorre em até $20 \%$ dos casos. A mama esquerda é mais acometida. Seu diagnóstico geralmente é clínico, no entanto, suas características e comportamento clínico são imprevisíveis. Diante disso, o diagnóstico é estabelecido por meio de mamografia, ultrassonografia mamária e biópsia. O tratamento baseia-se na cirurgia, dependendo da extensão, tipo histológico e diferenciação do tumor. Objetivo: realizar uma análise da literatura vigente acerca do tumor filoide maligno com componente sarcomatoso, levando em consideração a importância do diagnóstico precoce correto, possíveis métodos diagnósticos, identificar as limitações e chances de sucesso e fatores que podem agravar o quadro. Metodologia: Trata-se de uma revisão integrativa da literatura, onde se realizaram a seleção e a identificação da bibliografia dentro da temática alvo, utilizando-se artigos científicos em bases de dados publicados entre os anos de 2015 e 2018. Como base para a realização do estudo, foram selecionados sete

\footnotetext{
${ }^{1}$ Enfermeira pós graduanda em Nefrologia.

${ }^{2}$ Enfermeira graduada pela Faculdade Santa Maria - FSM.

3 Docente-Faculdade Santa Maria. Enfermeiro-Faculdade Santa Maria. Especialista em Gestão do cuidado com ênfase no apoio matricial-Universidade Federal da Paraíba. Especialista em preceptoria no SUS - Instituto de Ensino e Pesquisa Hospital Sírio Libanês. Mestre em Saúde ColetivaUniversidade Católica de Santos.

${ }^{4}$ Doutora em Ciências pela Faculdade de Medicina do ABC. Especialista em Fisioterapia em Terapia Intensiva pela Associação Brasileira de Fisioterapia Cardiorespiratoria e Fisioterapia em Terapia Intensiva - ASSOBRAFIR. Especialista em Saúde Pública pela Faculdade de Ciências Sociais Aplicadas - FACISA. Docente da Faculdade Santa Maria.

${ }_{5}^{5}$ Graduada em Enfermagem - FAZER; Licenciada em Enfermagem - UFPB; Especialista em Auditoria em Serviços de Saúde - FACISA; Mestre em enfermagem - UFPB, Doutora em Ciências da Saúde pela Faculdade de Medicina do ABC - FMABC, Docente da Faculdade Santa Maria.
} 
artigos, encontrados nas bases de dados SciELO, LILACS e PubMED, onde um trata-se de um estudo retrospectivo, um estudo quantitativo, duas revisões integrativas e três estudos de caso. Ressalta-se a carência de artigos em português abordando a temática em questão. Conclusões: diante do exposto, constata-se que a fragilidade no diagnóstico, pela indisponibilidade de uma rede de atenção e de equipamentos de saúde, predispõe a um pior prognóstico, especialmente pelo retraso no diagnóstico, que, quando precoce, influi positivamente para celeridade no tratamento e melhoria do prognóstico.

Palavras chaves: Câncer de mama. Componente sarcomatoso. Filoide.

ABSTRACT: Introduction: The filoid tumor (FT) of the breast is a fibroepithelial neoplasm, responsible for less than $1 \%$ of breast tumors. It is the only benign breast tumor in $80 \%$ of cases capable of malignancy, which occurs in up to $20 \%$ of cases. The left breast is more affected. Its diagnosis is usually clinical, but its characteristics and clinical behavior are unpredictable. Therefore, the diagnosis is established through mammography, breast ultrasound and biopsy. Treatment is based on surgery, depending on the extent, histological type and differentiation of the tumor. Objective: To perform an analysis of the current literature on malignant phyloid tumor with sarcomatous component, taking into account the importance of correct early diagnosis, possible diagnostic methods, identifying limitations and chances of success and factors that may aggravate the condition. Methodology: This is an integrative literature review, in which the bibliography was selected and identified within the target theme, using scientific articles in databases published between 2015 and 2018. Seven articles from the SciELO, LILACS, and PubMED databases were selected, of which one is a retrospective study, one quantitative study, two integrative reviews and three case studies. It is important to highlight the lack of articles in Portuguese addressing the theme in question. Conclusions: The fragility in the diagnosis, due to the unavailability of a care network and health equipment, predisposes to a worse prognosis, especially due to the delayed diagnosis, which, when early, positively influences the rapidity of the diagnosis. treatment and prognosis improvement.

Keywords: Breast neoplasms. Sarcomatous component. Filoid. 


\section{INTRODUÇÃO}

O tumor filoide (TF) da mama é uma neoplasia fibroepitelial, responsável por menos de $1 \%$ dos tumores de mama. Apesar de raro, pode acometer mulheres de todas as idades, tornando importante o reconhecimento do tumor Filoide. Entretanto, tem sua maior incidência em mulheres com idade entre 35 e 55 anos, e somente em $3 \%$ dos casos ocorrem acometimento bilateral e acomete raramente o sexo masculino (RODRIGUES et al., 2014; SILVA et al., 2013).

Além disso, o TF é o único tumor benigno da mama em $80 \%$ dos casos, capaz de malignar-se, o que ocorre em até $20 \%$ dos casos. Apresenta alta tendência de recidiva local e pode sofrer degeneração maligna sarcomatosa e acometer gânglios axilares inflamatórios em $17 \%$ e metastáticos em cerca de 1\%. Entretanto, mesmo quando maligno, o TF apresenta baixa taxa disseminação sistêmica e, quando ocorre, afeta mais fígado, pulmão, osso e cérebro (RODRIGUES et al.,2014; SILVA et al., 2013).

O tumor tem superfície lisa, que raramente envolve a área do mamilo, é móvel, bem delimitado. A mama esquerda é mais acometida que a direita. Ao exame físico, apresenta-se, geralmente, com grande extensão, como uma massa, e a paciente relata um crescimento rápido e indolor (MELO et al., 2015; CARVALHO, 2011).

Seu diagnóstico geralmente é clínico, no entanto, suas características e comportamento clínico são imprevisíveis, tornando a clínica insuficiente para determinar um tratamento e seu diagnóstico pré-operatório. A mamografia não define o diagnóstico e a punção aspirativa com agulha fina e a biópsia percutânea com agulha grossa têm baixo valor preditivo. No entanto, a biópsia com agulha grossa (core biopsy) pode diferenciar o tumor filoide do carcinoma, não discriminando a variedade benigna da maligna, havendo, portanto, a necessidade de proceder com o exame anatomopatológico, fundamental ao diagnóstico, e podendo contribuir para a terapêutica (NAZARIO et al., 2007; MELO et al., 2015). 
Então, de acordo com suas características anatomopatológicas do componente mesenquimatoso, podem ser classificados em benignos (70\%), borderline $(7 \%)$ e malignos (23\%). Existe, portanto, uma dificuldade em seu diagnóstico, em função do rápido crescimento, grande tamanho, e apresentar aparência semelhante ao fibroadenoma. Diante disso, o diagnóstico é estabelecido por meio de mamografia, ultrassonografia mamária e biópsia (NUNES et al., 2014; BONFIM et al., 2014).

O tratamento baseia-se na cirurgia, dependendo da extensão, tipo histológico e diferenciação do tumor, pode ser tumorectomia, estendendo-se à mastectomia em casos de tumores borderline, malignos ou recorrentes. Para evitar recidivas locais, a completa excisão do tumor, com margens cirúrgicas livres, é provavelmente o fator isolado mais importante (MOREIRA; SALES; SANTOS, 2012; RODRIGUES et al., 2014).

Nunes et al. (2014) salientam que, se após o procedimento cirúrgico, as margens de segurança forem insuficientes, é recomendada a re-excisão com margens cirúrgicas livres para os tumores filoides borderline e malignos. Não é conveniente o esvaziamento axilar, uma vez que o envolvimento ganglionar é muito raro.

De acordo com Bonfim et al. (2014), conforme o seu comportamento clínico e prognóstico, os tumores devem ser tratados como sarcomas de mama primário, e não como carcinoma ductal infiltrativo. A radioterapia não tem sido utilizada nesse tipo de tumor, sendo a quimioterapia útil para tumores metastáticos, devendo ser utilizados protocolos para sarcoma (NUNES et al.,2014).

A motivação para o trabalho surgiu do conhecimento dos indicadores epidemiológicos, que reforçam a pertinência deste trabalho e sua importância com ênfase no seu manejo devido a sua raridade e difícil diagnóstico, objetivando, assim, conhecer a importância de um diagnóstico precocemente correto, identificando os possíveis métodos diagnósticos, limitações, chances de sucesso e os fatores que podem prejudicar.

Assim, tem-se por objetivo realizar uma análise da literatura vigente acerca do tumor filoide maligno com componente sarcomatoso, levando em consideração a importância do diagnóstico precoce correto, possíveis métodos diagnósticos, 
identificar as limitações e chances de sucesso e fatores que podem agravar o quadro.

\section{METODOLOGIA}

Trata-se de uma revisão integrativa da literatura, cujos dados foram obtidos a partir da análise de estudos já existentes na literatura vigente.

Inicialmente, realizaram-se a seleção e a identificação da bibliografia dentro da temática alvo, utilizando-se artigos científicos em bases de dados publicados entre os anos de 2015 e 2018, a partir de pesquisas listadas na base de dados da Biblioteca Virtual em Saúde (BVS), utilizando os DeCS "tumor filoide", "sarcomatoso", "tumor de mama". Os critérios para a inclusão foram: estudos que abordem a temática proposta, no idioma português e inglês, estarem dentro do recorte de tempo proposto, resumo e texto completo e disponível de forma online; e os critérios de exclusão foram publicações do tipo tese, jornais, resenhas ou que não se enquadrem em quaisquer dos quesitos de inclusão. A pesquisa das publicações foi realizada nos meses de Junho a Outubro de 2019.

Foram identificadas publicações nas bases de dados: Literatura LatinoAmericano e do Caribe em Ciências da Saúde (LILACS), Medical Literature Analysis and Retrieval System (MEDLINE), Scientific Electronic Library Online (SciELO), BDENF e PUBMED.

Mediante uma seleção e leitura minuciosa de todos os artigos encontrados nas bases de dados pesquisadas, foram selecionados sete artigos que se enquadravam nos critérios de inclusão propostos. 


\section{RESULTADOS}

$\mathrm{Na}$ tabela 1, estão contidos os sete artigos selecionados para o desenvolvimento do estudo, divididos em quatro variáveis.

Tabela 1: Distribuição dos artigos selecionados.

\begin{tabular}{|c|c|c|c|}
\hline $\begin{array}{c}\text { AUTORES / ANO } \\
\text { DE } \\
\text { PUBLICAÇÃO } \\
\end{array}$ & TÍTULO & PERIÓDICO/LOCAL & DELINEAMENTO \\
\hline $\begin{array}{l}\text { MATOS et al. } \\
(2017)\end{array}$ & $\begin{array}{l}\text { Tumor filoides de mama: } \\
\text { avaliação retrospectiva de } \\
\text { casos do Hospital do } \\
\text { Servidor Público Estadual } \\
\text { de São Paulo. }\end{array}$ & $\begin{array}{l}\text { Mastology } \\
\text { (Impr.) }\end{array}$ & $\begin{array}{l}\text { Estudo } \\
\text { retrospectivo }\end{array}$ \\
\hline $\begin{array}{l}\text { GATALICA et } \\
\text { al. }(2016)\end{array}$ & $\begin{array}{lr}\text { Multiplatform } & \text { molecular } \\
\text { profiling } & \text { identifies } \\
\text { potentially } & \text { targetable } \\
\text { biomarkers in malignant } \\
\text { phyllodes tumors of the } \\
\text { breast. }\end{array}$ & Oncotarget & $\begin{array}{l}\text { Estudo } \\
\text { quantitativo }\end{array}$ \\
\hline $\begin{array}{l}\text { EL OCHI et al. } \\
(2016)\end{array}$ & $\begin{array}{l}\text { Bone metastasis from } \\
\text { malignant phyllodes breast } \\
\text { tumor: report of two cases. }\end{array}$ & BMC Clin Pathol & Estudo de caso \\
\hline $\begin{array}{l}\text { LIGHTNER et } \\
\text { al. (2015) }\end{array}$ & $\begin{array}{l}\text { A single-center experience } \\
\text { and review of the literature: } \\
64 \text { cases of phyllodes } \\
\text { tumors to better } \\
\text { understand risk factors and } \\
\text { disease management. }\end{array}$ & $\begin{array}{l}\text { The American } \\
\text { Surgeon }\end{array}$ & $\begin{array}{l}\text { Revisão } \\
\text { Integrativa }\end{array}$ \\
\hline $\begin{array}{l}\text { NARLA et al. } \\
(2018)\end{array}$ & $\begin{array}{l}\text { Well-differentiated } \\
\text { liposarcoma of the breast } \\
\text { arising in a background of } \\
\text { malignant phyllodes tumor } \\
\text { in a pregnant woman: } A \\
\text { rare case report and } \\
\text { review of literature. }\end{array}$ & $\begin{array}{l}\text { Indian Journal of } \\
\text { Pathology and } \\
\text { Microbiology }\end{array}$ & $\begin{array}{l}\text { Revisão } \\
\text { Integrativa }\end{array}$ \\
\hline $\begin{array}{l}\text { WHITSELL et } \\
\text { al. (2018) }\end{array}$ & $\begin{array}{l}\text { High-grade pleomorphic } \\
\text { liposarcoma of the breast. }\end{array}$ & $\begin{array}{ll}\text { Grand } & \text { Rounds } \\
\text { Journal } & \end{array}$ & Estudo de caso. \\
\hline $\begin{array}{l}\text { SINGER } \\
\text { al.(2018) }\end{array}$ & $\begin{array}{l}\text { Unusual aggressive breast } \\
\text { cancer: } \quad \text { metastatic } \\
\text { malignant phyllodes tumor. }\end{array}$ & $\begin{array}{l}\text { Journal } \\
\text { radiology } \\
\text { reports }\end{array}$ & Estudo de caso. \\
\hline
\end{tabular}

Fonte: Dados da pesquisa, 2019. 
Foram selecionados sete artigos, sendo um da SciELO, um da LILACS e cinco da PubMED. Ressalta-se a carência de artigos em português abordando a temática em questão.

Dentre os artigos selecionados, um trata-se de um estudo retrospectivo, um estudo quantitativo, duas revisões integrativas e três estudos de caso. Os artigos do tipo estudo de caso obtiveram maioria perante os demais tipos de delineamentos. Este tipo de estudo trata-se de pesquisas realizadas a partir de experiências vivenciadas pelos próprios autores, endossadas com apoio metodológico (LUKOSEVICIUS et al., 2018).

As contribuições são:

- $\quad$ MATOS et al. (2017): o estudo foi desenvolvido a partir dos dados de 52 pacientes com tumor filoide, onde se analisaram-se as características das pacientes e um quantitativo de recidivas das mesmas. Na análise, observou-se que a idade média das portadoras do tumor é de 45 anos e, em relação ao tratamento, 12 foram submetidas à mastectomia e 40 , à extirpação total. Houve 17 recidivas após cerca de 53,93 meses, achados que corroboram outras literaturas.

- GATALICA et al. (2016): apresentam que, dependendo das características do componente sarcomatoso, este tumor pode exibir um espectro morfológico entre os feno-genomas e os sarcomas estromais puros $A$ apresentação morfológica é composta de células estromais com formato de fuso e são fibroblásticas ou miofibroblásticas. Entretanto, esta morfologia inclui diferenciação óssea benigna e maligna, condróide, adiposa, rabdomioblástica e do músculo liso, sendo mais de um tipo de diferenciação que pode ser visto dentro do componente sarcomatoso Este pode desenvolver supercrescimentos significativos com um elemento bifásico residual mínimo que só pode ser observado após a exaustiva amostragem. A incidência de lipossarcoma nas mamas varia de $6 \%$ a $24 \%$.

- $\quad$ EL OCHI et al. (2016): discorrem que os lipossarcomas têm predileção pelas extremidades inferiores, cintura pélvica e retroperitônio. Outras localizações 
raras incluem cavidade peritoneal, axila, vulva, cordão espermático e mama. Existem poucos relatos de casos de lipossarcomas surgindo em um contexto de tumor filoide, no entanto, a apresentação maligna com desdiferenciação em si é uma entidade rara, com poucas séries de casos relatados no mundo. O diagnóstico diferencial na neoplasia com componente sarcomatoso na mama inclui lipossarcoma primário, secundário e lipoma.

- LIGHTNER et al. (2015): o estudo endossa que, apesar de as características clínicas não serem específicas e variarem entre a localização das metástases ósseas desta neoplasia, os exames de imagem, como radiografias e tomografia computadorizada, podem mostrar uma massa sólida adjacente ao osso envolvido e infiltrando-se no córtex e na medula em um padrão permeativo; já a ressonância magnética pode delinear melhor a extensão metastática e descrição do tumor. Além disso, o exame anatomopatológico é caracterizado por uma proliferação maligna de fascículos de células fusiformes, com atipia nuclear e alto índice mitótico sem componente epitelial. No entanto, a imuno-histoquímica demonstra apenas positividade à vimentina, os marcadores de pancitoqueratina, actina do músculo liso, desmina, proteína S100, CD34, C31, CD99 e CD117 são, geralmente, negativas. A positividade dos receptores de estrogênio e progesterona nunca havia sido relatada.

- $\quad$ NARLA et al. (2018): apontam que o tratamento para esta neoplasia com componente sarcomatoso é a excisão local ampla, com margens livres de pelo menos $2 \mathrm{~cm}$. Logo, a mastectomia é recomendada quando as margens estão envolvidas ou se há metástase nodal positiva.

- WHITSELL et al. (2018): a pesquisa apresenta que o uso de radioterapia e quimioterapia adjuvante pode ser limitado a pacientes com margens cirúrgicas positivas, metástases distantes são vistas em 10 a $20 \%$ dos casos, podendo ocorrer mesmo após cirurgia inicial de mama tecnicamente adequada. Os fatores preditivos mais confiáveis para o desenvolvimento de metástases distantes são crescimento excessivo do estroma, pleomorfismo nuclear e alta atividade mitótica, sendo o tamanho do tumor e recorrência local é controverso. A sobrevida 
livre de recidiva foi de 0,8 e 0,47 aos 1 e 3 anos no primeiro caso, 0,76 e 0,4 aos 1 e 3 anos no segundo caso.

- SINGER et al. (2018): a pesquisa endossa que os achados morfológicos e imuno-histoquímicos são de fundamental importância na exclusão de sarcomas, mioepitelioma, carcinoma sarcomatoide metastático, melanoma e tumor estromal gastrointestinal, pois é difícil fazer um diagnóstico específico apenas por exame microscópico, o diagnóstico de confirmação deve ser baseado na correlação clínico e anatomopatológica

\section{CONCLUSÃO}

O diagnóstico correto da diferenciação celular, molecular e morfológica requer a identificação do componente bifásico do tumor, além de ser realizado diagnóstico diferencial com outras categorias. Por isso, faz-se necessária uma investigação precisa das metástases, que deve ser baseada na correlação clínica e anatomopatológica, permitindo a exclusão de diagnósticos diferenciais, sendo fundamentais a detecção precoce e a ressecção completa antes da disseminação para tratamento com êxito e bom prognóstico da paciente.

Diante do exposto, constata-se que a fragilidade no diagnóstico, pela indisponibilidade de uma rede de atenção e de equipamentos de saúde, predispõe a um pior prognóstico, especialmente pelo retraso no diagnóstico que, quando precoce, influi positivamente para celeridade no tratamento e melhoria do prognóstico.

Ressalta-se que este tipo de estudo é de suma importância para a realização de novos estudos a fim de aprofundamento no assunto, visto que é um tema pouco debatido e, até mesmo no meio científico, encontra-se pouco material a respeito. 


\section{REFERÊNCIAS BIBLIOGRÁFICAS}

AL-RABIY, F.N.; ALI, R.H. Malignant phyllodes tumor with osteosarcomatous differentiation metastasizing to small bowel and causing intestinal obstruction. Diagnostic Histopathology. v. 21, n. $4, \quad$ p. $165-168, \quad 2015 . \quad$ Disponivel em: <https://www.sciencedirect.com/science/article/abs/pii/S1756231714001467>. https://doi.org/10.1016/j.mpdhp.2014.09.001.

BONFIM, R. J. A. et al. Tumor filoide em adolescente: relato de caso em jovem de 13 anos. Rev Bras Mastologia. v. 23, n. 4, p. 135-140, 2013. Disponível em: <http://www.mastology.org/wpcontent/uploads/2015/06/MAS_v23n4_135-140.pdf>.

http://dx.doi.org/10.5327/Z2013000400008RM.

$\mathrm{EL} \mathrm{OCHI}, \mathrm{M}$. R. et al. Bone metastasis from malignant phyllodes breast tumor: report of two cases. BMC Clin Pathol. v. 16, n. 4, 2016. Disponível em: <https://bmcclinpathol.biomedcentral.com/track/pdf/10.1186/s12907-016-0027-7>.

https://doi.org/10.1186/s12907-016-0027-7.

GATALICA, Z. et al. Multiplatform molecular profiling identifies potentially targetable biomarkers in malignant phyllodes tumors of the breast. Oncotarget. v. 7, n. 2, p.1707-1716, 2016. Disponivel em: <https://www.ncbi.nlm.nih.gov/pmc/articles/PMC4811491/>. https://dx.doi.org/10.18632\%2Foncotarget.6421

GIL, A. C. Métodos e técnicas de pesquisa social. São Paulo: Atlas, 1999.

$\begin{array}{ll}\begin{array}{l}\text { INSTITUTO BRASILEIRO DE GEOGRAFIA E ESTATÍSTICA. PAS - Pesquisa Anual de } \\ \text { Serviços, }\end{array} & \text { Disponível } \\ 2017 . & \text { em: }\end{array}$ $\begin{array}{llr}\text { Serviços, } & \text { 2017. } & \text { Disponível } \\ \text { <https://cidades.ibge.gov.br/xtras/perfil.php?lang=\&codmun=211223\&search=maranhao|trizidela- }\end{array}$ do-vale|infograficos:-informacoes-completas>. Acesso em: 15 out. 2017.

LUKOSEVICIUS, A. P.; GUIMARÃES, J. C. Uso do Método Estudo de Caso em Pesquisas de Gerenciamento de Projetos. Revista de Gestão e Projetos. v. 9, n. 2, p.20-35, 2018. Disponível em: $\quad<h t t p: / / w w w . r e v i s t a g e p . o r g / o j s / i n d e x . p h p / g e p / a r t i c l e / v i e w / 656 / p d f>$. https://dx.doi.org/10.5585/gep.v9i2.656.

LIGHTNER, A. L. et al. A single-center experience and review of the literature: 64 cases of phyllodes tumors to better understand risk factors and disease management. Am. Surg. v. 81, n. 3, p. 309-315, 2015. Disponível em: <https://www.ncbi.nlm.nih.gov/pubmed/25760210>.

MATOS, A. B. T. M. B. et al. Phyllodes tumors of the breast: a retrospective evaluation of cases from the Hospital do Servidor Público Estadual de São Paulo. Mastology. v. 27, n. 4, p.339-343, 2017. Disponível em: <https://pesquisa.bvsalud.org/portal/resource/pt/biblio-884322>.

MELO, C. P. et al. Tumor filoides de mama: relato de caso. In: MOSTRA DE PRODUÇÃO UNIVERSITÁRIA, 14. 2015, Rio Grande. Rio Grande: Universidade Federal do Rio Grande do Sul, 2015.

MOREIRA, K. C.; SALES, W. T.; SANTOS, E. M. Tumor filoides maligno com diferenciação para lipossarcoma pleomórfico na mama. Rev. Bras. Mastologia. v. 22, n. 2, p. 66-69, 2012. Disponível em: <http://www.mastology.org/wp-content/uploads/2015/06/MAS_v22n2_66-69.pdf>.

NARLA, S. L. et al. Well-differentiated liposarcoma of the breast arising in a background of malignant phyllodes tumor in a pregnant woman: A rare case report and review of literature. Indian J Pathol Microbiol. v. 61, n. 4 , p. 577-579, 2018. Disponível em: 
$<$ http://www.ijpmonline.org/article.asp?issn=0377-

4929; year $=2018$; volume $=61$; issue $=4 ;$ spage $=577$; epage $=579$; aulast $=$ Narla $>$.

https://doi.org/10.4103/IJPM.IJPM_238_17

PEREIRA, L. T. K.; GODOY, D. M. A.; TERÇARIOL, D. Estudo de caso como procedimento de pesquisa científica: reflexão a partir da clínica fonoaudiológica. Psicol. Reflex. Crit. v. 22, n. 3, p. 422-429, 2009. Disponível em: <http://www.scielo.br/scielo.php?script=sci_arttext\&pid=S010279722009000300013\&lng=en\&nrm=iso >. https://dx.doi.org/10.1590/S0102-79722009000300013

RODRIGUES, G. C. R. et al. Tumor filoide maligno de mama bilateral: relato de caso. Rev. Bras.

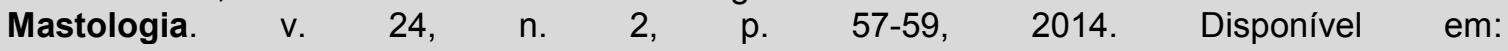
<https://pesquisa.bvsalud.org/portal/resource/pt/lil-781041?lang=es>.

ROWE, J. J.; PRAYSON, R. A. Metastatic malignant phyllodes tumor involving the cerebellum. Journal of Clinical Neuroscience. v. 22, n. 1, p. 226-227, 2015. Disponível em: <https://www.sciencedirect.com/science/article/abs/pii/S0967586814005694>.

https://doi.org/10.1016/j.jocn.2014.09.004.

SILVA, K. F. P. et al. Phyllodes breast tumor: case series and review of literature. Rev. Bras. Mastologia. v. 22, n. 2, p. 42-45, 2013. Disponível em: <http://www.mastology.org/wpcontent/uploads/2015/06/MAS_v22n2_42-45.pdf>.

SINGER, A. et al. Unusual aggressive breast cancer: metastatic malignant phyllodes tumor. J Radiol Case Rep. v. 7, n. 2, p. 24-37, 2013. Disponível em: $<$ https://www.ncbi.nlm.nih.gov/pmc/articles/PMC3661309/>.

https://dx.doi.org/10.3941\%2Fjrcr.v7i2.1430

SOUSA, E. D. P. de; SANTOS FILHO, A. B. dos; DIAS, C. P. Sarcoma de mama: relato de caso. Rev. Bras. Mastologia. v. 21, n. 2, p. 78-80, 2011. Disponível em: <http://www.mastology.org/wp-content/uploads/2015/06/MAS_v21n2_78-80.pdf>.

VENTURA, M. M. O estudo de caso como modalidade de pesquisa. Rev SoCERJ. v. 20, n. 5, p. 383-386, 2007.

WHITSELL, T. et al. High-grade pleomorphic liposarcoma of the breast. Grand Rounds Journal. v. $11, \quad$ p. $\quad 87-91, \quad 2011 . \quad$ Disponível em: < https://pdfs.semanticscholar.org/9391/dbe9c45e9f344c4ded42e1420ded027cbd1e.pdf>. https://dx.doi.org/10.1102/1470-5206.2011.0022

YOSHIDA, W.B. Redação do relato de caso. J. vasc. bras. v. 6, n. 2, p. 112-113, 2007. Disponível em: <http://www.scielo.br/scielo.php?script=sci_arttext\&pid=S167754492007000200004>. https://dx.doi.org/10.1590/S1677-54492007000200004.

ZAMUDIO, J. L.; LEMUS, D. Z.; SOTO, P. A. O. Breast myxofibrosarcoma. Case report and literature review. Rev Mex Mastol. v. 7, n. 1, p. 11-14, 2017. Disponível em: <https://www.medigraphic.com/pdfs/revmexmastol/ma-2017/ma171c.pdf>. 